\title{
AN INEQUALITY FOR SELFADJOINT OPERATORS ON A HILBERT SPACE
}

\author{
HERBERT J. BERNSTEIN
}

\begin{abstract}
An elementary inequality of use in testing convergence of eigenvector calculations is proven. If $e_{\lambda}$ is a unit eigenvector corresponding to an eigenvalue $\lambda$ of a selfadjoint operator $A$ on a Hilbert space $H$, then

$$
\left|\left(g, e_{\lambda}\right)\right|^{2} \leq \frac{\|g\|^{2}\|A g\|^{2}-(g, A g)^{2}}{\|(A-\lambda I) g\|^{2}}
$$

for all $g$ in $H$ for which $A g \neq \lambda g$. Equality holds only when the component of $g$ orthogonal to $e_{\lambda}$ is also an eigenvector of $A$.
\end{abstract}

When computing eigenvectors of real symmetric matrices by iterative techniques, convergence is usually assumed on the basis of stagnation of Rayleigh quotients. (e.g., see [2]). In most cases this is satisfactory. However, when dealing with pathologically close eigenvalues, significant components orthogonal to the desired vector may remain $[3$, p. 174]. In such cases one may try to estimate the size of those orthogonal components to decide if, say, Richardson's purificatin is needed. In this paper we present an estimator which has been of value in such calculations. The proof is elementary and the author suspects that it is not new, but is able to find no prior publication nor use within standard eigenvector programs.

Before proving the inequality, we state a lemma which can be derived directly from the equations used in proving Cauchy's or Schwartz' inequality $[1$, p. 16]. We include an abstract proof here for completeness.

LEMMA. Let $H$ be a Hilbert space, $A$ a selfadjoint operator on $H, x$ a vector in $H$, and let $\tau$ be real. Then

$$
\|x\|^{2}\|A x\|^{2}-(x, A x)^{2}=\|x\|^{2}\|(A-\tau I) x\|^{2}-(x,(A-\tau I) x)^{2} .
$$

PROOF.

$$
\begin{aligned}
\|x\|^{2} \| & (A-\tau I) x \|^{2}-(x,(A-\tau I) x)^{2} \\
= & \|x\|^{2}\left(\|A x\|^{2}-2 \tau(x, A x)+\tau^{2}\|x\|^{2}\right) \\
& -\left((x, A x)^{2}-2 \tau(x, A x)\|x\|^{2}+\tau^{2}\|x\|^{4}\right) \\
= & \|x\|^{2}\|A x\|^{2}-2 \tau(x, A x)\|x\|^{2}+\tau^{2}\|x\|^{4} \\
& -(x, A x)^{2}+2 \tau(x, A x)\|x\|^{2}-\tau^{2}\|x\|^{4} \\
= & \|x\|^{2}\|A x\|^{2}-(x, A x)^{2} \text {. Q.E.D. }
\end{aligned}
$$

Now we can prove the desired inequality.

Received by the editors January 27, 1986.

1980 Mathematics Subject Classification (1985 Revision). Primary 65F15; Secondary 65J10.

This research was partially supported by the Department of Energy under contract DEACO276ERO3077-V, the National Science Foundation, under contract DCR-8511302, and the Office of Naval Research, under contract N00014-82-K-0381. 
THEOREM 1. Let $H$ be a Hilbert space, $A$ a selfadjoint operator on $H, e_{\lambda} a$ unit eigenvector of $A$ with corresponding eigenvalue $\lambda$, and let $f$ be orthogonal to $A$. Then for any $g=\alpha e_{\lambda}+f$ with $\alpha$ real, either $A g=\lambda g$ or

$$
\alpha^{2} \leq \frac{\|g\|^{2}\|A g\|^{2}-(g, A g)^{2}}{\|(A-\lambda I) g\|^{2}} .
$$

ProOF. By the lemma, we may replace $A$ in the numerator by $A-\lambda I$, allowing us to assume $\lambda=0$ without loss of generality. However, in that case

$$
A g=A f, \quad(g, A g)=(f, A f), \quad \text { and } \quad\|A g\|=\|A f\|
$$

so that

$$
\begin{aligned}
\frac{\|g\|^{2}\|A g\|^{2}-(g, A g)^{2}}{\|A g\|^{2}} & =\frac{\left(\alpha^{2}+\|f\|^{2}\right)\|A f\|^{2}-(f, A f)^{2}}{\|A f\|^{2}} \\
& =\alpha^{2}+\frac{\|f\|^{2}\|A f\|^{2}-(f, A f)^{2}}{\|A f\|^{2}} \geq \alpha^{2}
\end{aligned}
$$

by the Schwartz inequality. Q.E.D.

Notice that the inequality is strict unless the Schwartz inequality on $(f, A f)$ is an equality, i.e., when $f$ is an eigenvector of $A$. It then follows immediately that

COROLLARY. If $e_{\lambda}$ is a unit eigenvector corresponding to eigenvalue $\lambda$ of a selfadjoint operator $A$ on a Hilbert space $H$, then

$$
\left|\left(g, e_{\lambda}\right)\right|^{2} \leq \frac{\|g\|^{2}\|A g\|^{2}-(g, A g)^{2}}{\|(A-\lambda I) g\|^{2}}
$$

for all $g$ in $H$ for which $A g \neq \lambda g$. Equality will hold if and only if the component of $g$ orthogonal to $e_{\lambda}$ is also an eigenvector of $A$.

We now show that the bound given is actually the best of the bounds that could be obtained from a class of squares of ratios of shifted inner products to the eigenvalue shifted by the same amount.

THEOREM 2. Under the hypotheses of Theorem 1, let $\tau$ be real. Then either $A g=\lambda g$ or

$$
\frac{\|g\|^{2}\|A g\|^{2}-(g, A g)^{2}}{\|(A-\lambda I) g\|^{2}} \leq \frac{\|(A-\tau I) g\|^{2}}{(\lambda-\tau)^{2}} .
$$

Proof. Since $a / b \leq c / d$ for $b, d>0$ if and only if $b c-a d \geq 0$, we define $B=A-\lambda I$ and, using the lemma, compute

$$
\begin{aligned}
&\|(A-\lambda I) g\|^{2}\|(A-\tau I) g\|^{2}-(\lambda-\tau)^{2}\left(\|g\|^{2}\|A g\|^{2}-(g, A g)^{2}\right) \\
&=\|B g\|^{2}\|(B+(\lambda-\tau) I) g\|^{2}-(\lambda-\tau)^{2}\left(\|g\|^{2}\|B g\|^{2}-(g, B g)^{2}\right) \\
&=\|B g\|^{4}+2(\lambda-\tau)\|B g\|^{2}+(\lambda-\tau)^{2}\|g\|^{2}\|B g\|^{2} \\
&-(\lambda-\tau)^{2}\|g\|^{2}\|B g\|^{2}+(\lambda-\tau)^{2}(g, B g)^{2} \\
&=\left(\|B g\|^{2}+(\lambda-\tau)(g, B g)\right)^{2} \geq 0
\end{aligned}
$$

with equality only when $(g,(A-\lambda I) g) \neq 0$ and

$$
\tau=\lambda+\frac{\|(A-\lambda I) g\|^{2}}{(g,(A,-\lambda I) g)} \text {. Q.E.D. }
$$




\section{REFERENCES}

1. G. H. Hardy, J. E. Littlewood, and G. Pólya, Inequalities, Cambridge Univ. Press, Cambridge, 1934.

2. H. Rutishauser, Simultaneous iteration method for symmetric matrices, Numer. Math. 16 (1970), 205-223.

3. J. H. Wilkinson, The algebraic eigenvalue problem, Clarendon Press, Oxford, 1965.

Courant InStitute of Mathematical Sciences, NeW YORK UNIVERSity, NeW YORK, NEW YORK 10012 